\title{
Wireless Point-of-Care Platform With Screen-Printed Sensors for Biomarkers Detection
}

\author{
Sarah Tonello, Giulia Abate, Michela Borghetti, Mariagrazia Marziano, Mauro Serpelloni, Member, IEEE, \\ Daniela L. Uberti, Nicola Francesco Lopomo, Maurizio Memo and Emilio Sardini, Member, IEEE
}

\begin{abstract}
Measurement systems for early and reliable detection of degenerative diseases, such as Alzheimer's disease (AD), are extremely important in clinical diagnosis. Among these, biochemical assays represent a commonly used method to distinguish patients from healthy population thanks to the sensitive recognition of specific biomarkers in biological fluids. In order to overcome actual limitations of these techniques in term of cost, standardization, and sensitivity, this study aimed to realize a low-cost highly sensitive portable point-of-care (PoC) testing system based on screen-printed electrochemical sensors. The development of the platform specifically included both the design of the sensing probe and the electronic circuit devoted to condition and acquires the transduced electric signal. The designed circuit was implemented in a printed circuit board and interfaced to a wireless system based on bluetooth data transmission in order to improve the portability of the proposed solution. Preliminary results were obtained by using controlled concentrations of electrolytic solutions and calibrating the sensors for antibodies and for a well-known protein (i.e., interleukin 8) quantified by anodic stripping voltammetry (ASV). Findings from ASV measurements showed a sensitivity of $38 \mu \mathrm{A} /(\mathrm{ng} / \mathrm{ml})$ with a tested range from 1.25 to $20 \mathrm{ng} / \mathrm{ml}$, with a limit of detection of $2 \mathrm{ng} / \mathrm{ml}$. Further investigation will include the validation of this PoC device by testing the concentration of a specific p53 protein isoform, which was recently identified to early correlate to AD development.
\end{abstract}

Index Terms-Electrochemical biosensors, point-of-care (PoC) testing, screen printing, voltammetry, wireless data acquisition device.

\section{INTRODUCTION}

$\mathbf{R}$ ECENTLY, one of the most pervasive challenges of electronics and engineering applied to biotechnological and clinical research has been represented by the ability to develop specific measurement devices with integrated technologies able to identify-quickly, diffusely, and with a very high sensitivity-specific disease-related proteins and biomolecules, defined as "biomarkers." In this perspective, new rapid, low cost and easily accessible methodologies and

Manuscript received September 28, 2016; revised March 21, 2017; accepted March 23, 2017. The Associate Editor coordinating the review process was Dr. Salvatore Baglio. (Corresponding author: Mauro Serpelloni.)

S. Tonello, M. Borghetti, M. Serpelloni, N. F. Lopomo, and E. Sardini are with the Department of Information Engineering, University of Brescia, 25123 Brescia, Italy (e-mail: s.tonello@unibs.it; mauro.serpelloni@ing.unibs.it).

G. Abate, M. Marziano, D. L. Uberti, and M. Memo are with the Department of Molecular and Translational Medicine, University of Brescia, 25123 Brescia, Italy.

Color versions of one or more of the figures in this paper are available online at http://ieeexplore.iee.org.

Digital Object Identifier 10.1109/TIM.2017.2692308 technologies have been increasingly investigated-including medical devices for in vitro diagnosis and point-of-care $(\mathrm{PoC})$ systems-supported also by the growing interest toward customized and personalized medicine and toward rapid and home-accessible portable diagnostic systems [1]-[3].

Portable and self-standing devices, fundamental for $\mathrm{PoC}$ applications, can be realized by integrating proper sensing devices with conditioning and acquisition circuit [1].

Different technologies can be adopted in order to realize biosensors integrated in lab-on-a-chip devices, promising path to reduce time, cost, and quantity of sample required to perform the analysis [2]. Furthermore, user safety and sample sterility can also be addressed by implementing wireless module, as highlighted in [3].

Among the different techniques investigated, electrochemical biosensors represent the ideal starting point to realize complete testing platforms for biological biomarker quantification. More specifically, screen-printed electrochemical sensors (SPES), thanks to their ability to be functionalized and customized for the detection of different analytes (e.g., DNA, proteins), have acquired a predominant importance in the last decade. Printed electronics presents a successful approach to develop low-cost solutions, with the possibility to increase the level of standardization, to reduce sample volumes required for each analysis and to customize the surface for a more efficient functionalization. In particular, literature reports different studies based on screen printing (also defined as thick film printing) to realize electrochemical sensors addressing the sensitive and specific quantification of different proteins and biomolecules [4], [5].

Electrochemistry offers a wide variety of techniques to detect and quantify specific biomolecules using SPES, including the possibility to both completely immerse the sensor in a buffer solution [6] or just cover it with a small volume of sample [7]. All the electrochemical approaches could be very fundamental in PoC implementation, since they can associate an improved quantitative analysis, with the possibility to maintain a wet setup with functionalization, using a protocol similar to the one usually implemented for enzyme-linked immunosorbent assays (ELISA).

According to the signal recorded, these techniques can be divided into impedance based and voltammetry methods. Among the first group, electrochemical impedance spectroscopy (EIS) represents a label-free method useful for protein and biomolecules quantification, particularly 
interesting due to its ability to detect variations in resistance and capacitance upon binding events. EIS principle is based on the measurement of the changes of impedance deriving from a different electrons exchange at the interface between the functionalized surface of the electrode and a conductive solution. Considering the same setup, these changes are in particular related only to the concentration of the recognized proteins [9]. Differently from EIS, in all the voltammetric techniques, information about the analyte is obtained by measuring the current as the potential of the electrode is varied. Even if with differences between the specific biomolecules, detection is performed labeling target analytes with selected redox reactive species, thus to correlate an electrical signal proportional to the amount of analyte recognized. Among voltammetric methods, anodic stripping voltammetry (ASV) represents one of the most promising compared to any other electrochemical techniques, able to increase of sensitivity of others 2 or 3 orders of magnitude, reaching limit of detection (LOD) in the range of $10^{-9}$ to $10^{-10} \mathrm{M}$. In ASV, a metal is deposited on the surface of the electrodes during a preconcentration step, and it is then stripped applying an oxidizing potential. In addition to heavy metals quantification, interesting applications of ASV for proteins [7], biomolecules [11], Deoxyribonucleic acid (DNA) sequences [12] have been investigated. Labeling proteins with specific catalyzing enzymes, proteins concentration can be indirectly quantified as proportional to the amount of metal deposited by measuring current peak metal during the stripping step.

Similarly, for both EIS and voltammetry, a three-electrode configuration is usually implemented. This configuration, including a working, counter and reference electrode (Working Electrode (WE), Counter Electrodes (CE), and Reference Electrode (RE)), is most common for typical electrochemical applications, especially when the potential across the electrochemical interface need to be measured accurately, in order to ensure stability of the cell [8].

Concerning the integration of the conditioning circuit in the design of PoC systems or monitoring devices, we can find different examples in the very recent literature, which tried to improve different clinical issues related to rapidity of assays, portability, implantability, and sensitivity. More specifically in order to allow an efficient transduction of physiological processes into quantifiable signal, combinations of potentiostats and galvanostats standard designs have been investigated in different solutions [13]. Additionally, a great interest was related to combine innovative detection techniques (including label-free techniques based on optical, mechanical and electrochemical transducers) with their optimal conditioning circuit in order to realize fast, portable, and easily usable devices aiming to smart bio sensing and to bring a significant improvement to clinical daily practice [3], [14]. However, among the various technologies proposed for the realization of $\mathrm{PoC}$ systems, no reliable, quantitative, and sensitive devices for specific protein detection have been yet commercialized.

Therefore, our specific aim is to apply these challenging up-and-coming $\mathrm{PoC}$ concept, extending previous preliminary analyses [15], in order to realize a self-standing portable device, which could be useful for the sensitive quantification of protein in biomarkers detection.

In particular, the interest of this paper concerned the realization of a platform for biomarkers involved in the early diagnosis of Alzheimer's disease (AD). Among neurodegenerative diseases, one of the most pervasive challenges is in fact related to the ability to detect AD in its earliest stage [16], by using different strategies, including cognitive tests, neuroimaging [17], [18], genetic profiling and biomarkers. Peripheral AD biomarkers in particular have recently attracted attention for their minimally invasive approach [19], stimulating the discussion and investigation on different novel techniques. Specifically, a peculiar expression of an altered conformational isoform of p53 protein was reported to be able to distinguish AD subjects from healthy population, demonstrating high values of sensitivity and specificity [20]. So far, quantification of this protein can be performed in clinical and biological laboratories by using a specific ELISA assay starting from peripheral blood samples. However, this technique presents issues in term of time and cost effectiveness, required sample volume, inter- and intrarater reliability, and possibility of quantification.

In this perspective, focusing on the quantification of biomarkers for early detection of $\mathrm{AD}$, but without losing the possibility of generalization, this paper aimed to complete and improve results obtained in previous preliminary analyses [15], introducing a system able to perform a sensitive and reliable analysis, with operator-friendly characteristic and wireless transmission of data aiming at improving portability.

Therefore, here is presented a complete system consisting of a SPES with six measuring points and an electronic microprocessor-based system, designed to wirelessly transmit data, thus to increase the overall portability of the solution. Particular attention was given to the choice of the SPES materials and geometry, the electronic circuit devoted to the conditioning and acquisition of the electric signal, and to the electronic microprocessor-based solution that can transmit data wirelessly. Several characterization measurements were also obtained with the presented system and further design considerations and critical analyses are reported.

\section{System Description, Materials, And Methods}

The proposed PoC is composed by three main parts (Fig. 1): 1) the screen-printed sensing probe, which can be functionalized using different antibodies for the detection of specific biomarkers; 2) the signal conditioning circuit designed to ensure highly precise and sensitive measurements, thus to register small changes in ionic current during ASV testing protocols; and 3) the wireless Bluetooth system for signal acquisition.

\section{A. Sensors Design and Production}

Sensor layout was designed using a software called QCAD (QCAD.org). Each layer, corresponding to a different conducting material, was separately designed, in order to produce the masks required to screen print-layer-by-layerthe final structure of the sensor. Conductive tracks were designed with particular care using a resolution compatible 


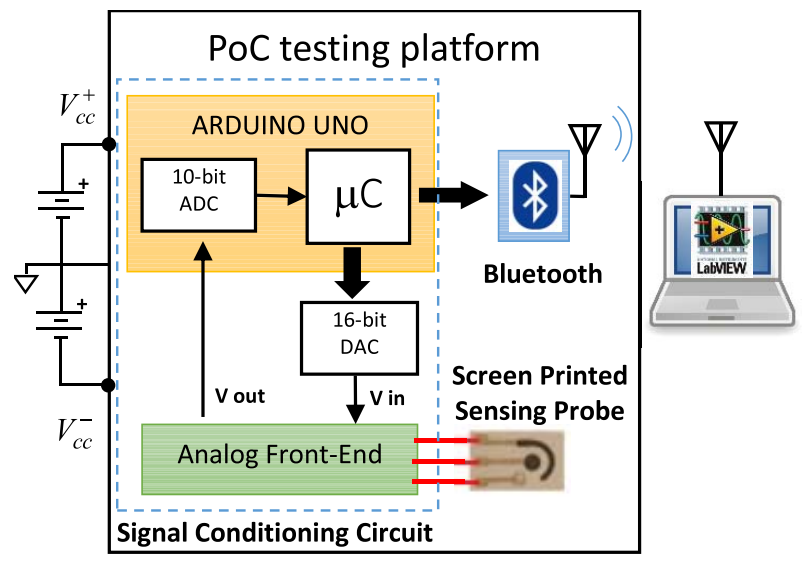

Fig. 1. Schematic of the proposed PoC testing platform.

with the adopted screen printer (A2 Model, Baccini srl, Italy). The geometry-with WEs of $5 \mathrm{~mm}$ of diameter-was optimized from a single to a multiwell design. This specific configuration allows reducing variability, improving the performances when testing duplicates or different biomarkers during same analysis. Following a standard procedure, final geometry was printed on a lucid sheet by means of inkjet printing, thus to allow the realization by UV photolithography of a $40-\mu$ m-thick blocking stencil, i.e., the mask required for the screen printing process. Specifically, a silver ink (CI 1001, electrical resistance $<0.015 \mathrm{ohms} /$ square, viscosity $14 \mathrm{~Pa} \mathrm{~s}$ at $30{ }^{\circ} \mathrm{C}$ ) was selected to realize the conductive tracks. Carbon ink was selected to realize the WE and $\mathrm{CE}$ of the biosensor. Among the materials available for SPES production (e.g., carbon, gold, platinum), carbon represents the best compromise in term of cost-essential requirement when designing a PoC device-biocompatibility-in order to ensure a proper functionalization-and electronic performances - in order to allow signal transduction during ASV measurements [21]. Finally, a silver-silver chloride ink (CI 4002, electrical resistance $<0.050 \Omega^{-2}$, viscosity $5 \mathrm{~Pa}$ $\mathrm{s}$ at $30{ }^{\circ} \mathrm{C}$ ) thanks to its inert chemical composition, was selected to realize the RE. All the conductive pastes were purchased from ECM-Engineered Conductive Materials (Engineered Material System Inc.). Using a screen with 250 meshes with apertures forming angles of $45^{\circ}$, the three layers were consequently printed on a $0.4-\mathrm{mm}$-thick alumina substrate: first silver, then carbon and finally silver-silver chloride. Each layer was cured in an oven, specifically silver inks for $10 \mathrm{~min}$ at $110{ }^{\circ} \mathrm{C}$ and carbon ink $5 \mathrm{~min}$ at $130{ }^{\circ} \mathrm{C}$. After the printing process was completed, in order to allow a better conduction of the signal, the conductive tracks were covered by using a transparent spray (RS Components, U.K.) specifically adopted in printed electronics as a protecting layer to ensure proper electrical insulation. This operation only leaves the terminal part of the tracks uncoated, thus to allow the connection with the conditioning circuit (Fig. 2).

\section{B. Circuit Design and Production}

Following the completion of the SPES final layout, a dedicated acquisition system was designed in order to allow the production of a complete PoC testing platform.
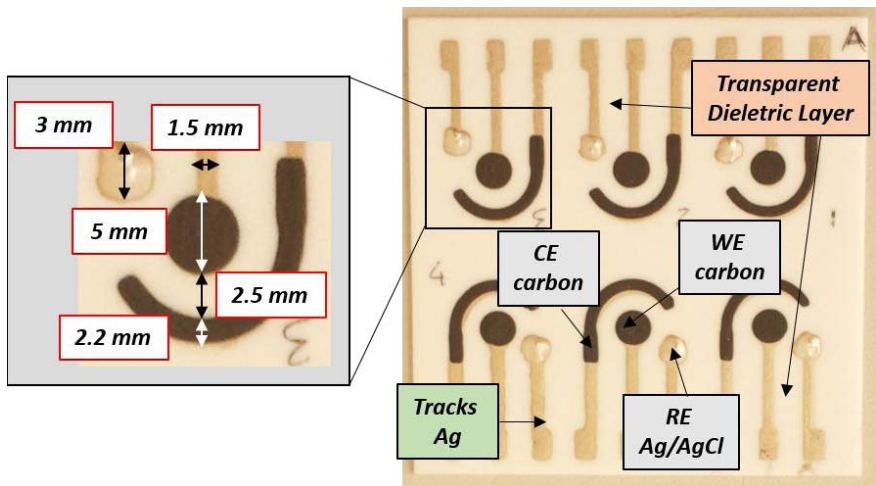

Fig. 2. Final SPES layout.

The testing solution is basically composed of one Arduino UNO board, a custom conditioning circuit and a bluetooth module. For the dual-voltage supply $( \pm 5 \mathrm{~V})$, two lithiumion batteries were adopted. Arduino UNO board generates the signal required to drive the measurement. Since Arduino UNO is not able to generate an analog signal, it communicates with the 16-b digital-to-analog converter (16-b DAC) over serial peripheral interface in order to obtain an analog signal $\left(V_{\text {in }}\right)$, as input for the conditioning circuit. The design of the analog front end was performed to obtain ASV measurements (Fig. 3). In particular, the desired potential is applied to the cell by controlling the potential of RE with respect to the WE and, thanks to the electrons movement generated by the effect of the electromotive force applied in the sample, the generated current can be detected between WE and CEs. The last stage was developed to make the output of the OPA129 suitable for the Arduino analog input. Indeed, the OPA129 output can be positive or negative while the analog input of Arduino can be only positive. Operational amplifiers were carefully selected depending on the specific function they had to perform in the circuit. In order to guarantee a high accuracy in the control of the input voltage to the sensor, precision bipolar amplifiers were selected [OPA177GS from Texas Instrument (TI)], with very low offset voltage, drift, and low noise. In order to allow low-current measurements with high precision and low bias, the current-to-voltage converter was realized using an ultralow bias current monolithic operational amplifier (OPA129 from TI).

Once set the maximum values of the voltage $\left(V_{\max , \mathrm{RE}}\right)$ required for elicit specific electrochemical reaction, $V_{\text {in }}$ was imposed as reported in Fig. 3 and $V_{\text {out }}$ was then measured within the interval $\Delta \mathrm{T}$, which specifically depends on both $V_{\text {max.RE }}$ and the overall scan cycle duration $(T)$. The scan rate is defined as the ratio between maximum voltage $\left(V_{\max , \mathrm{RE}}\right)$ and the time required to reach this voltage $(T / 4)$.

After all the Surface Mounting Device (SMD) components were soldered, the board was inserted in a metallic box to avoid noises on the signal recording and to improve the sensor sensitivity and precision. The output signal $\left(V_{\text {out }}\right)$ is acquired by Arduino UNO through the internal 10$\mathrm{b}$ analog-to-digital converter. Measurements are transmitted wirelessly to a PC using the Bluetooth module. The transmitted packet includes the time, $V_{\text {in }}$ and $V_{\text {out }}$ values, A Virtual Instrument (VI) was developed to acquire and store the data. Furthermore, through the VI the user can define 


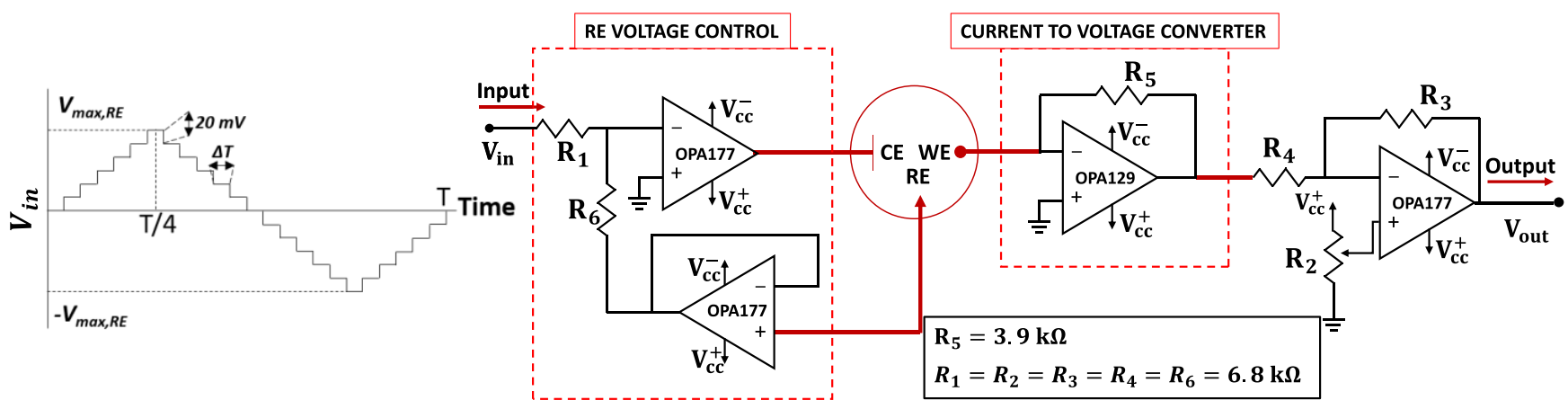

Fig. 3. Schematic of the waveform of $V_{\text {in }}$ produced by DAC (Left) and schematic of the circuit used as analog front end (Right).

the $V_{\max . R E}$ and the $T$ when the setup code of Arduino UNO is executed. In this way, with the possibility of generalize this approach, the PoC could be used for identifying several biomarkers, by implementing different SPESs and functionalization methods. Overall dimension of the proposed $\mathrm{PoC}$ device is defined by the specific dimension of each of its components: sensor $5.1 \mathrm{~cm} \times 5.1 \mathrm{~cm}$ (with a specific active area of each WE of $20 \mathrm{~mm}^{2}$, Fig. 2), PCB $7.6 \mathrm{~cm} \times 3.3 \mathrm{~cm}$, and bluetooth module with Arduino $7.5 \mathrm{~cm} \times 7 \mathrm{~cm} \times 4.5 \mathrm{~cm}$.

\section{Calibration and Measurements}

After optimizing the design and testing the compatibility of the materials with wet lab techniques, the platform was assessed by measuring output current, following three different protocols: 1) using a saline solution, with different concentrations of $\mathrm{NaCl}$; 2) functionalizing the WE with primary antibodies in presence of $\mathrm{K}_{3}\left[\mathrm{Fe}(\mathrm{CN})_{6}\right]$ solution; and 3) performing ASV measurements to calibrate the platform using Interleukin 8 (IL-8).

Both the input and the output signals were generated and acquired respectively by using the portable wireless system described in the previous paragraph. Experiments were always performed in triplicate. All graphical and tabulated data were usually reported as mean \pm mean standard error. LOD for IL-8 quantification was calculated using three-sigma limit definition.

1) $\mathrm{NaCl}$ Solution Measurements: The first test of the circuit was performed in order to assess its ability to detect small changes in solution conductivity due to controlled variations of $\mathrm{NaCl}$ concentration in deionized (DI) water. Specifically the circuit was evaluated with concentrations lower than $1 \mathrm{mg} / \mathrm{ml}$ : $0,0.44,0.66,0.88$, and $1 \mathrm{mg} / \mathrm{ml}$. In each experiment, drops of $200 \mu 1$ of saline solution were released on the three electrodes, ensuring that the drop stayed correctly in place by using a mask applied on top of the sensor. For this analysis, $V_{\max , \mathrm{RE}}$ and $T$ were set to $300 \mathrm{mV}$ and $25 \mathrm{~s}$, respectively, and thus the scan rate was $48 \mathrm{mV} / \mathrm{s}$. The expected output was related to a change in the intensity of current flowing between WE and $\mathrm{CE}$, which corresponds to a different concentration.

2) Antibodies Coating Quantification: The effectiveness of primary antibodies coating on the WE was assessed by evaluating changes in the current detected between WE and $\mathrm{CE}$ at different scan rates with three antibodies concentrations:

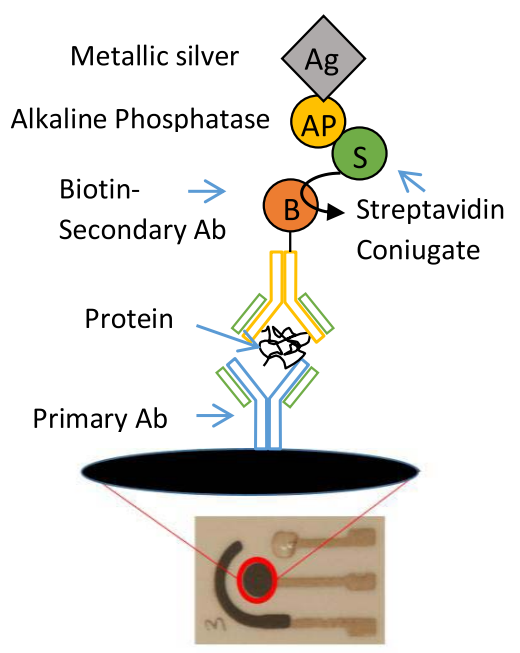

Fig. 4. Sensor functionalization for ASV measurements.

0,4 , and $8 \mu \mathrm{g} / \mathrm{ml}$. After an overnight incubation at $4{ }^{\circ} \mathrm{C}$, the measurements were performed in presence of a conductive electrolytic solution of $5 \mathrm{mM} \mathrm{K}_{3}\left[\mathrm{Fe}(\mathrm{CN})_{6}\right]$ in 1-M KCl. Once the functionalization was performed, a drop of $200 \mu \mathrm{l}$ was placed in order to cover the electrodes and allow current flow. Finally, the electronic measurements were performed at four different scan rates $(48,120,240$, and $1.2 \mathrm{mV} / \mathrm{s})$, with a $V_{\text {max,RE }}$ of $300 \mathrm{mV}$.

3) Interleukin Quantification Using ASV: In order to optimize the ASV protocol for biomarkers quantification (Fig. 4), a preliminary curve was quantified using IL-8 protein, a member of the CXC chemokine subfamily. Cytokines are important mediators of inflammation and are usually associated with pathogenesis of many inflammatory diseases. Therefore, IL-8 is currently being applied as noninvasive diagnostic marker in various fields of medicine either for the purpose of early diagnosis or as a prognosis predictor [22]. Furthermore, recently, Ray et al. [23] has identified 18 signaling proteins in blood plasma that can be used to classify and predict clinical Alzheimer's diagnosis, among which IL-8 has been also identified. Therefore, IL-8 can be considered as universal biomarkers-from cancer and inflammation to neurodegeneration-thus generalizing the use of the present PoC platform to various clinical fields. 


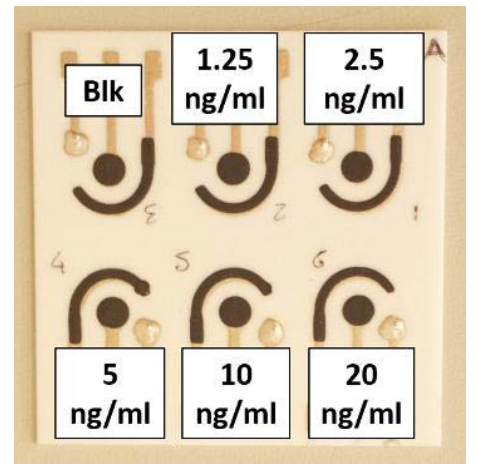

Fig. 5. Layout for IL-8 concentration test on multiwells sensor.

Moreover, in this validation phase, IL-8 strong interaction with its capture antibody, allows to reduce the variability to the functionalization phase. The protocol adopted for IL-8 quantification in this calibration phase of the circuit was characterized by a specific functionalization of the sensor using immunocomplexes formed by a capture and a detection antibody, using a dedicated kit (DuoSet development system for ELISA, Human CXCL8/IL-8). In particular, during the functionalization step different concentration of IL-8 were dropped on the multiwell sensor, as shown in Fig. 5.

In order to evaluate sensor LOD of IL-8, the platform was calibrated using a range of concentrations usually easily quantifiable using with ELISA assay, from 1.25 to $20 \mathrm{ng} / \mathrm{ml}$. In order to perform the detection step, the functionalization was completed labeling the secondary antibodies with alkaline phosphatase, an enzyme able to catalyze the reaction from ionic silver of silver nitrate $\left(\mathrm{AgNO}_{3}\right)$ to metallic silver, allowing a selective deposition of silver, proportional to the amount of protein recognized in the sample.

Thus, Ascorbic acid (AA-p) $+2 \mathrm{Ag}$ reacts in Dehydroascorbic acid $+2 \mathrm{H}+2 \mathrm{Ag}$. After the deposition step was completed, in order to perform the stripping step, $200 \mu \mathrm{l}$ of buffer solution $(1-\mathrm{M} \mathrm{KCl})$ was dropped on the three electrodes to allow current flow and $V_{\max , \mathrm{RE}}$ and $T$ were set, respectively, to $900 \mathrm{mV}$ and $60 \mathrm{~s}$, thus with a scan rate of $60 \mathrm{mV} / \mathrm{s}$. Finally, current flowing between $\mathrm{WE}$ and $\mathrm{CE}$ due to $\mathrm{Ag}$ oxidation was measured, allowing a sensitive quantification of proteins recognized on the sensor surface.

\section{RESULTS}

\section{A. Calibration and Measurements}

1) $\mathrm{NaCl}$ Solution Measurements: Results from the evaluation of circuit response to changes in saline solution conductivity showed a linear increase of current peaks corresponding to increasing $\mathrm{NaCl}$ concentrations. This finding was coherent with literature since, as expected, the conductivity of a specific solution has a positive linear relationship with its concentration [24]. In particular, in the considered range of concentrations, the circuit showed the ability to detect small changes in solution conductivity corresponding to small values of current flowing between $\mathrm{WE}$ and $\mathrm{CE}$, thus reporting a sensitivity of $0.15 \mathrm{~mA} /(\mathrm{mg} / \mathrm{ml})$, with a current of $0.07 \mathrm{~mA}$ at zero $\mathrm{NaCl}$ concentration, due to DI water conductivity (Fig. 6).

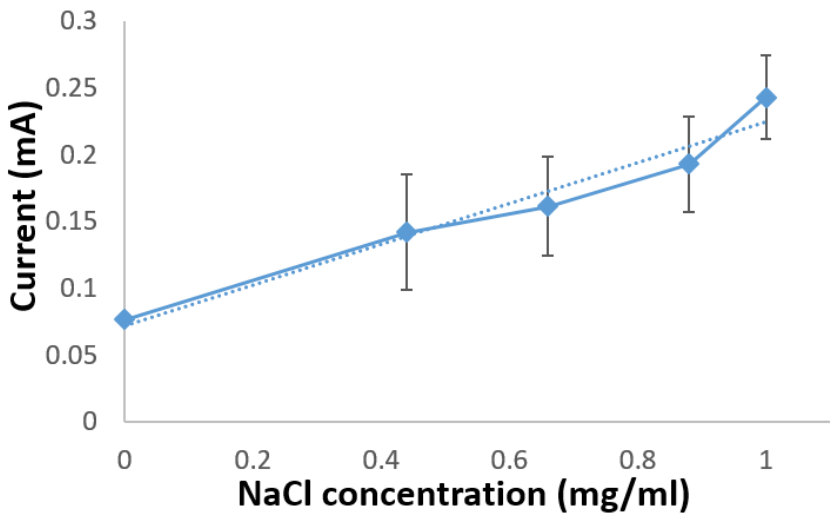

Fig. 6. Local maximum of the voltammograms peaks at different concentrations of IL-8.

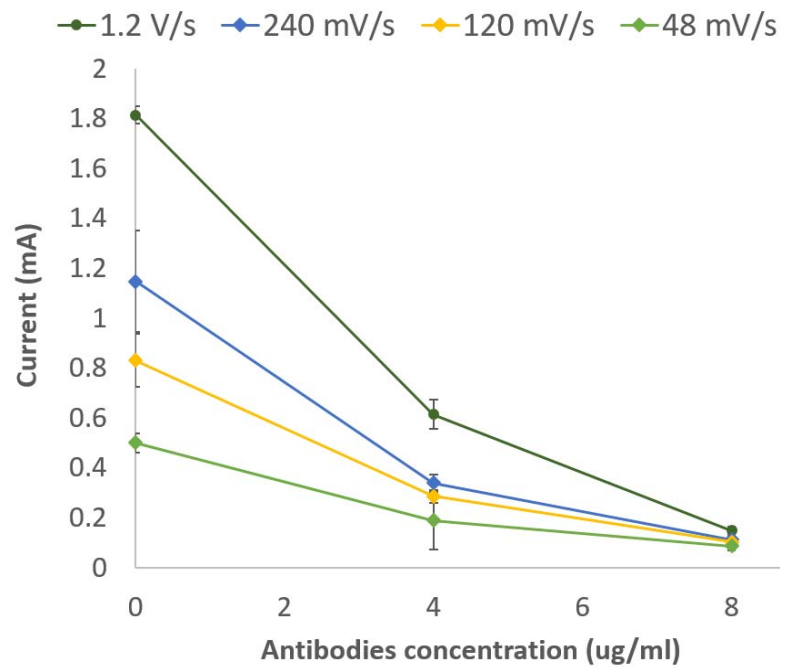

Fig. 7. Calibration of SPES with $\mathrm{NaCl}$ solution. (Error bars represent standard deviations of measured data.)

2) Antibodies Coating Quantification: Results from antibodies coating quantification showed a proportional decrease of the current peak flowing between WE and CE, for all the scan rates evaluated, indicating an increased impedance of the system due to an increasing concentration of antibodies coated on WE surfaces (Fig. 7).

This behavior comes from an increased portion of WE covered with primary antibodies, and, therefore, from a reduced conductive surface of the electrode available to exchange electrons with the electrolytic solution.

The sensitivity of the platform to quantify the concentration of antibodies coated on the WE appears to be affected from the scan rate of the input signal. In particular, a sensitivity of $52 \mu \mathrm{A} / \mu \mathrm{g}$ was measured at $48 \mathrm{mV} / \mathrm{s}$, of $90 \mu \mathrm{A} / \mu \mathrm{g}$ at $120 \mathrm{mV} / \mathrm{s}$, of $128 \mu \mathrm{A} / \mu \mathrm{g}$ at $240 \mathrm{mV} / \mathrm{s}$, and of $208 \mu \mathrm{A} / \mu \mathrm{g}$ could be calculated for measurements performed at $1.2 \mathrm{~V} / \mathrm{s}$.

Comparing the variation observed between the current peaks recorded using different scan rates, a more enhanced difference could be observed at higher scan rates. Even though in all the evaluated conditions an increase in the current peak recorded can be observed when decreasing the antibodies concentration. 


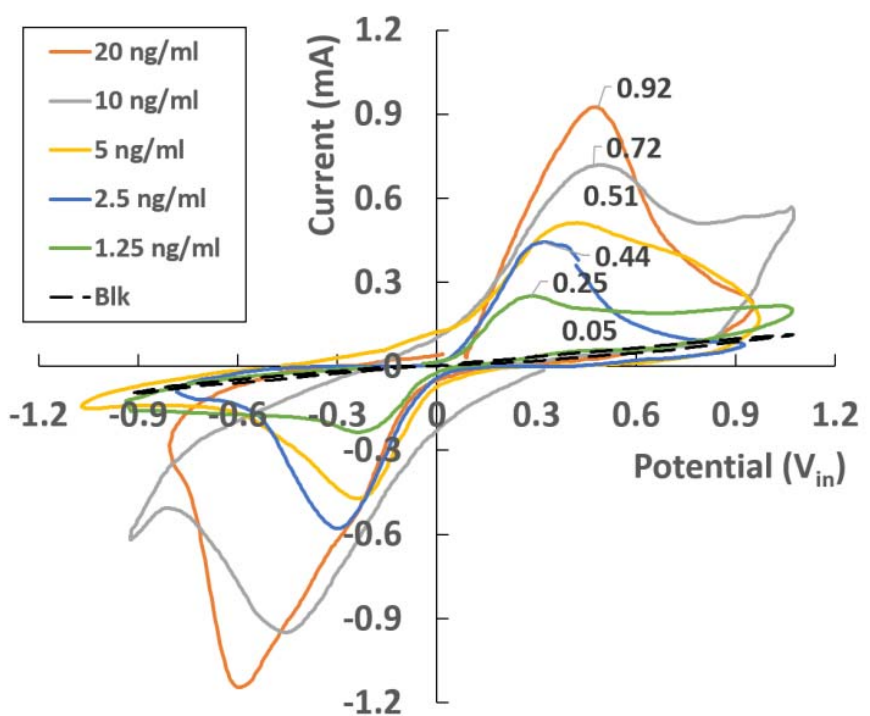

Fig. 8. Differences between antibodies concentration detection at different scan rates. (Error bars represent standard deviations of measured data.)

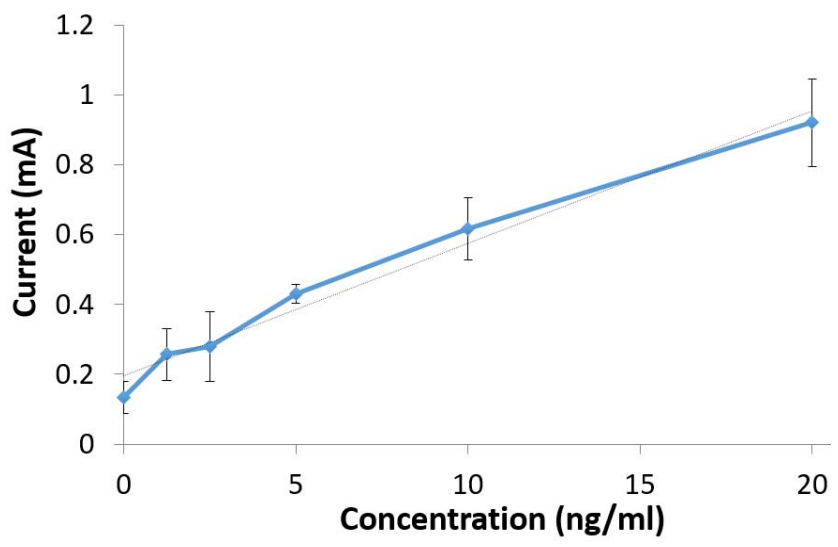

Fig. 9. Cyclic voltammograms, corresponding to different IL-8 concentrations.

For the bare sensor, where the entire surface is available for electrons exchange with the electrolytic solution, an enhanced difference between current recorded at different scan rates could be observed, compared with the coated conditions. This result suggested a good adhesion of the coated layer on the WE, acting as an insulating layer.

3) Interleukin Quantification Using ASV: Results from ASV measurements showed an increase in the maximum peak of current proportional to the increase of IL-8 concentration. The voltammograms plotted in Fig. 8 showed the anodic peak due to the silver stripped in the buffer solution in a window of positive potentials around $0.5 \mathrm{~V}$, corresponding to the oxidation potential of metallic silver deposited during the chemical deposition step. Bare electrodes (dotted black line), just coated with $8 \mu \mathrm{g} / \mathrm{ml}$ of primary antibody, showed a CV with a current peak corresponding to $0.05 \mathrm{~mA}$, value comparable with the results obtained from antibodies quantification considering a similar scan rate, as described in the previous paragraph. Some differences could be observed between the baselines corresponding to different electrodes and affecting the position of the peaks, probably due to intrinsic variability in the electrodes processing. Furthermore, also the local reaction between the electrode and the buffer solution could influence the baseline and may need to be analyzed further, in order to improve the reliability of the final PoC. The local maximum of the current peaks was calculated for each concentrations and then plotted (Fig. 9).

Well defined current peaks could be observed for the tested range of concentration from 1.25 to $20 \mathrm{ng} / \mathrm{ml}$, with a sensitivity of $38 \mu \mathrm{A} /(\mathrm{ng} / \mathrm{ml})$ and a LOD of $2 \mathrm{ng} / \mathrm{ml}$. The choice of the concentration tested in this phase form 1.25 to $20 \mathrm{ng} / \mathrm{ml}$ had to take into account the limitation due to the technique and the material (i.e., carbon ink) used to produce the WE. However, these values are in accordance with the range adopted in different works using ELISA assay.

\section{DISCUSSION AND CONCLUSION}

The complexities and the heterogeneity associated with $\mathrm{AD}$ require high precision and sensitivity in the reliable detection and quantification of specific biomarkers. This approach could allow to obtain an early diagnosis of the disease in the presymptomatic phase. Furthermore, the identification of specific biological markers could provide additional information about the pathology development from both the biological and patho-clinical point of view [19], [25]. In order to achieve this goal efficiently, different characteristics of measuring methodology — such as rapidity, low cost, and ease of access and use-play a fundamental role. Furthermore, this need of quantification has been rapidly growing also considering the interest addressed toward personalized medicine and domiciliary diagnostics [1].

The proposed PoC platform presented high level of integration of different solutions, including SPES and wireless transmission, in order to obtain a low cost and portable but highly sensitive device.

From the characterization perspective, the results obtained, confirmed the compatibility of the screen-printed materials for the functionalization required for $\mathrm{AD}$ biomarkers detection and wet lab practices. Furthermore, tests performed both with $\mathrm{NaCl}$ solution and with $\mathrm{K}_{3}\left[\mathrm{Fe}(\mathrm{CN})_{6}\right]$ solution validated the ability of the designed circuit to effectively detect changes in solution conductivity and system impedance, related to the changes in the current flowing between WE and CE. Results from the characterization with conductive solutions suggested several properties. First of all, the ability of the circuit to detect low changes in concentration, with good reproducibility and reliable responses. Furthermore, the response recorded for the electrode coated with $8 \mu \mathrm{g} / \mathrm{ml}$ of antibody antiIL-8 at the different frequencies could confirm the good adhesion of the primary antibody on the WE, essential for the effective realization of the complete immunocomplex. Findings obtained from the calibration performed quantifying IL-8 protein, showed a sensitivity of $38 \mu \mathrm{A} /(\mathrm{ng} / \mathrm{ml})$ and a LOD of $2 \mathrm{ng} / \mathrm{ml}$. These results appear to be promising also compared with works where, using similar experiment designs and ASV as detection method, a limit for a reliable quantification of proteins was reported to be $10 \mathrm{ng} / \mathrm{ml}$ [7], and $2.2 \mathrm{ng} / \mathrm{ml}$ [26], respectively. Decreasing the concentration of IL-8, thus decreasing the amount of silver stripped, ASV peaks appeared to be shifted toward decreasing poten- 
tials, due to the interaction between buffer solution and electrode when redox reaction is limited.

In this research, we set up all the conditions in order to develop a PoC device with a patented antibody recognizing unfolded p53 and allowing the measurement of this biomarker in $\mathrm{AD}$ patients. After the validation, the proposed methodology and the platform designed will be optimized in order to be easily accessible for a routine automatized diagnosis technique in the clinical environment.

All this, with the aim to realize an innovative self-standing portable point-of-care test system, representing a low cost, easy to use, and highly precise platform able to support the validation of a promising putative early biomarker for AD.

\section{ACKNOWLEDGEMENTS}

This work was partially performed within BIOMANE project, funded by the University of Brescia.

\section{REFERENCES}

[1] A. P. Dhawan et al., "Current and future challenges in point-of-care technologies: A paradigm-shift in affordable global healthcare with personalized and preventive medicine," IEEE J. Transl. Eng. Health Med., vol. 3, pp. 1-10, 2015.

[2] S. Feng, L. E. Roseng, and T. Dong, "Quantitative detection of Escherichia coli and measurement of urinary tract infection diagnosis possibility by use of a portable, handheld sensor," in Proc. IEEE Int. Symp. Med. Meas. Appl. (MeMeA), May 2015, pp. 586-589.

[3] S. Rampazzi, G. Danese, F. Leporati, and F. Marabelli, "A localized surface plasmon resonance-based portable instrument for quick on-site biomolecular detection," IEEE Trans. Instrum. Meas., vol. 65, no. 2, pp. 317-327, Feb. 2016.

[4] A. M. Pernía, M. J. Prieto, I. C. Orille, J. A. Martín-Ramos, and A. Costa-García, "Development of optimized screen-printed immunosensors," IEEE Trans. Instrum. Meas., vol. 58, no. 7, pp. 2181-2188, Jul. 2009.

[5] R. A. S. Couto, J. L. F. C. Lima, and M. B. Quinaz, "Recent developments, characteristics and potential applications of screen-printed electrodes in pharmaceutical and biological analysis," Talanta, vol. 146, pp. 801-814, Jan. 2016.

[6] R. Elshafey, A. C. Tavares, M. Siaj, and M. Zourob, "Electrochemical impedance immunosensor based on gold nanoparticles-protein $\mathrm{G}$ for the detection of cancer marker epidermal growth factor receptor in human plasma and brain tissue," Biosensors Bioelectron., vol. 50, pp. 143-149, Dec. 2013.

[7] V. Escamilla-Gómez, D. Hernández-Santos, M. B. González-García, J. M. Pingarrón-Carrazón, and A. Costa-García, "Simultaneous detection of free and total prostate specific antigen on a screen-printed electrochemical dual sensor," Biosensors Bioelectron., vol. 24, no. 8, pp. 2678-2683, Apr. 2009.

[8] J. L. Hammond, N. Formisano, P. Estrela, S. Carrara, and J. Tkac, "Electrochemical biosensors and nanobiosensors," Essays Biochem., vol. 60, no. 1, pp. 69-80, Jun. 2016.

[9] J. Kang, A. T. Hussain, M. Catt, M. Trenell, B. Haggett, and E. H. Yu, "Electrochemical detection of non-esterified fatty acid by layer-by-layer assembled enzyme electrodes," Sens. Actuators B, Chem., vol. 190, pp. 535-541, Jan. 2014

[10] Y.-F. Liang, C.-Y. Huang, and B.-D. Liu, "A voltammetry potentiostat design for large dynamic range current measurement," in Proc. Int. Conf. Intell. Comput. Bio-Med. Instrum. (ICBMI), Dec. 2011, pp. 260-263.

[11] R. J. C. Brown and M. J. T. Milton, "Stripping voltammetry as a possible primary method for amount of substance," IEEE Trans. Instrum. Meas., vol. 56, no. 2, pp. 280-283, Apr. 2007.

[12] G. Martínez-Paredes, M. B. González-García, and A. Costa-García, "Genosensor for detection of four pneumoniae bacteria using gold nanostructured screen-printed carbon electrodes as transducers," Sens. Actuators B, Chem., vol. 149, no. 2, pp. 329-335, Aug. 2010.

[13] R. D. Beach, R. W. Conlan, M. C. Godwin, and F. Moussy, "Towards a miniature implantable in vivo telemetry monitoring system dynamically configurable as a potentiostat or galvanostat for two- and three-electrode biosensors," IEEE Trans. Instrum. Meas., vol. 54, no. 1, pp. 61-72, Feb. 2005
[14] L. Bissi, P. Placidi, and A. Scorzoni, "A configurable mixed-signal architecture for label-free smart biosensor applications," IEEE Trans. Instrum. Meas., vol. 58, no. 5, pp. 1333-1344, May 2009.

[15] S. Tonello, M. Serpelloni, N. F. Lopomo, E. Sardini, G. Abate, and D. L. Uberti, "Preliminary study of a low-cost point-of-care testing system using screen-printed biosensors: For early biomarkers detection related to Alzheimer disease," in Proc. IEEE Int. Symp. Med. Meas. Appl. (MeMeA), May 2016, pp. 1-6.

[16] Z. Svobodova et al., "Development of a magnetic immunosorbent for on-chip preconcentration of amyloid $\beta$ isoforms: Representatives of Alzheimer's disease biomarkers," Biomicrofluidics, vol. 6, no. 2, pp. 024126-1-024126-12, Jun. 2012.

[17] A. Faro, D. Giordano, C. Spampinato, S. Ullo, and A. Di Stefano, "Basal ganglia activity measurement by automatic 3-D striatum segmentation in SPECT images," IEEE Trans. Instrum. Meas., vol. 60, no. 10, pp. 3269-3280, Oct. 2011.

[18] S. Borromeo, J. A. Hernandez-Tamames, G. Luna, F. Machado, N. Malpica, and A. Toledano, "Objective assessment of olfactory function using functional magnetic resonance imaging (fMRI)," IEEE Trans. Instrum. Meas., vol. 59, no. 10, pp. 2602-2608, Oct. 2010.

[19] T. K. Khan and D. L. Alkon, "Peripheral biomarkers of Alzheimer's disease," J. Alzheimer's Disease, vol. 44, no. 3, pp. 729-744, 2015.

[20] L. Buizza et al., "Conformational altered p53 as an early marker of oxidative stress in Alzheimer's disease," PLOS ONE, vol. 7, no. 1, p. e29789, 2012.

[21] L. J. C. Jeuken, Structure and Modification of Electrode Materials for Protein Electrochemistry. Berlin, Germany: Springer, 2016, pp. 1-31.

[22] A. Shahzad, M. Knapp, I. Lang, and G. Köhler, "Interleukin 8 (IL-8)A universal biomarker?" Int. Arch. Med., vol. 3, p. 11, Jun. 2010.

[23] S. Ray et al., "Classification and prediction of clinical Alzheimer's diagnosis based on plasma signaling proteins," Nature Med., vol. 13, no. 11, pp. 1359-1362, Nov. 2007.

[24] G. M. Marion and K. L. Babcock, "Predicting specific conductance and salt concentration in dilute aqueous solutions," Soil Sci., vol. 122, no. 4, 1976.

[25] L. J. Thal et al., "The role of biomarkers in clinical trials for Alzheimer disease," Alzheimer Disease Assoc. Disorders, vol. 20, no. 1, pp. 6-15, 2006.

[26] Z.-P. Chen, Z.-F. Peng, J.-H. Jiang, X.-B. Zhang, G.-L. Shen, and R.-Q. Yu, "An electrochemical amplification immunoassay using biocatalytic metal deposition coupled with anodic stripping voltammetric detection," Sens. Actuators B, Chem., vol. 129, no. 1, pp. 146-151, Jan. 2008.

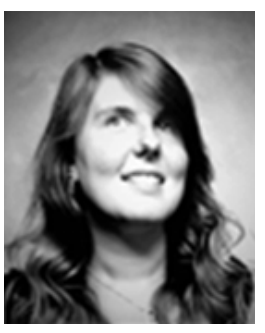

Sarah Tonello was born in Brescia, Italy, in 1990. She received the bachelor's (summa cum laude) degree in biomedical engineering from the Politecnico di Milano, Milan, Italy, in 2012, and the master's (summa cum laude) degrees in biomedical engineering from the University of Florida, Gainesville, FL, USA, and Politecnico di Milano, in 2014, under the Atlantis CRISP dual degree grant, with a focus on cell and tissue engineering and biotechnology. In 2014, she was enrolled in the Ph.D. Program of Technology for Health in the University of Brescia, Brescia.

She is currently with the Department of Information Engineering, University of Brescia. Her current research interests include printed sensors and electronic devices, electrochemical sensors, conductive scaffolds and biomaterials for applications in tissue engineering and regenerative medicine.

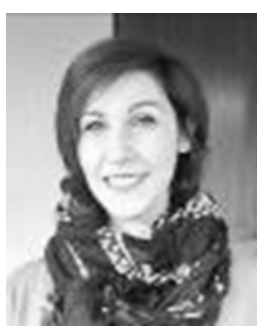

Giulia Abate was born in Montichiari, Brescia, Italy, in 1989. She received the bachelor's degree in biotechnology and the master's (summa cum laude) degree in medical biotechnology from the University of Brescia, Brescia, Italy, in 2012 and 2014, respectively, with a focus on the mitochondrial impairment and the redox unbalance involved in Alzheimer's disease. In 2014, she was enrolled in the Ph.D. Program in biomedical science and translational medicine curricula: neuroscience at the University of Brescia.

Her current research interests include the identification of new early predictive blood-biomarkers for Alzheimer's disease and nanotechnology-based biosensors for diagnostic application. 


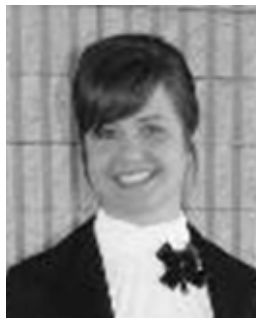

Michela Borghetti received the master's (cum laude) degree in electronic engineering and the Ph.D. degree in technology for Health from the University of Brescia, Brescia, Italy, in 2012 and 2016, respectively.

In 2015, she was a Visiting Ph.D. Student with the Universitat Politècnica de Catalunya, Barcelona, Spain. She is currently a Post-Doctoral Researcher with the Department of Information Engineering, University of Brescia. She is involved in the design and fabrication of sensors for healthcare using low-cost technologies, and also developing electronic systems for measuring and monitoring limb movements.

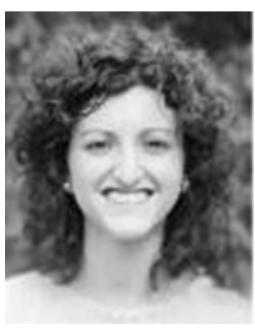

Mariagrazia Marziano was born in Brescia, Italy, in 1990. She received the bachelor's degree in biotechnology and the master's (summa cum laude) degree in medical biotechnology from the University of Brescia, Brescia, Italy, in 2012 and 2015, respectively, with a focus on neuroscience research and biotechnology. In 2015, she was enrolled in the $\mathrm{PhD}$ program of Technology for Health at the University of Brescia.

She is currently with the Department of Information Engineering and the Department of Molecular and Translational Medicine, University of Brescia. Her current research interests include printed sensors and proteomics approach with application in the field of Alzheimer's disease.

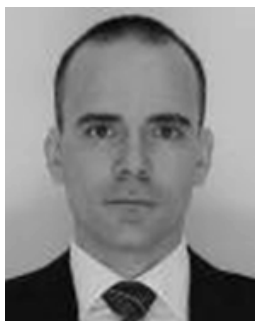

Mauro Serpelloni (M'12) was born in Brescia, Italy, in 1979. He received the M.S. (summa cum laude) degree in industrial management engineering and the Ph.D. degree in electronic instrumentation from the University of Brescia, Brescia, Italy, in 2003 and 2007, respectively.

$\mathrm{He}$ was involved in the design, modeling, and fabrication of measurement systems for industrial applications. He is currently an Assistant Professor of Electrical and Electronic Measurements with the Department of Information Engineering, University of Brescia. His current research interests include biomechatronic systems, contactless transmission between sensors and electronics, contactless activation for resonant sensors, and signal processing for microelectromechanical systems.

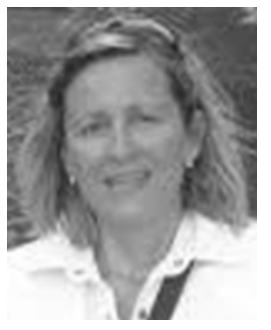

Daniela L. Uberti was born in Brescia, Italy. She received the bachelor's degree in chemistry and pharmaceutical technology from the University of Milan, Milan, Italy, in 1993, and the Ph.D. degree in neuroscience from the University of Brescia in 1999.

Over the past 15 years, she has focused on the early events occurring in periphery of Alzheimer's disease, with the aim to identify biomarkers, as risk factors for the development of the disease. Since 2004, she has been an Assistant Professor (Researcher) with the Department of Molecular and Translational Medicine, University of Brescia, Brescia, Italy. She is the Co-Owner of two patents: "Method for early identification of Alzheimer's Disease" (2003) 0001348902 and "Antibody binding a linear epitope of p53 and its diagnostic applications" PCT/EP2015/072094. She has authored or co-authored more than 60 peerreviewed publications.

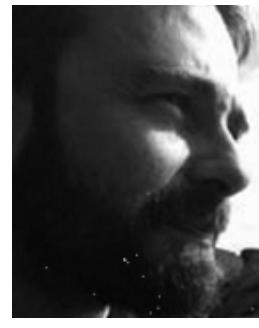

Nicola Francesco Lopomo received the M.S. degree in biomedical engineering and the Ph.D. degree in bioengineering from the Politecnico di Milano, Milan, Italy, in 2003 and 2008, respectively, defending with merits a thesis on computer-assisted surgery.

Since 2004, he has been a Research Fellow with the Laboratorio di Biomeccanica e Innovazione Tecnologica and Laboratorio di NanoBiotecnologie, Istituto Ortopedico Rizzoli, Bologna, Italy, where he was involved in musculoskeletal biomechanics and the deposition/characterization of ceramic coatings for orthopedic applications, respectively. Since 2015, he has been an Associate Professor of Bioengineering with the Dipartimento di Ingegneria dell'Informazione, Università degli Studi di Brescia, Brescia, Italy.

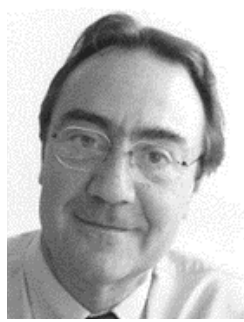

Maurizio Memo was born in Cagliari, Italy, in 1953. He received the bachelor's degree in chemistry and pharmaceutical technology from the University of Cagliari, Cagliari, Italy.

$\mathrm{He}$ is currently a Full Professor of Pharmacology with the University of Brescia, Brescia, Italy. He is the Director of Medical Pharmacology Specialization School and the Scientific Director of Drug Investigation, Information and Research, University Centre, University of Brescia. He is the Principal Investigator of several research projects sponsored by the National and International Institutions. He has authored more than 200 full scientific papers all published in recognized international Journals and the Organizer of 12 International Symposia.

Prof. Memo was a recipient of the Roussel Prize for Gerontological Research in 1987 and the Sandoz Foundation Prize in 1994.

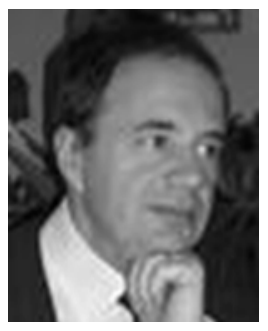

Emilio Sardini (M'99) was born in Commessaggio, Mantova, Italy, in 1958. He received the M.S. degree in electronics engineering from the Politecnico di Milano, Milan, Italy, in 1983.

Since 1984, he has been conducting research and teaching activities with the Department of Electronics for Automation, University of Brescia, Brescia, Italy, where he has been a Full Professor of Electrical and Electronic Measurements since 2006. He is currently the Coordinator of "Technology for Health" $\mathrm{Ph} . \mathrm{D}$. Program, the Director of the Department of Information Engineering, and a member of the Academic Senate with the University of Brescia. He has authored or co-authored more than 100 scientific papers. His research interests included electronic instrumentation, sensors, and signal conditioning electronics. 